\title{
Short Courses About Internet and Federal Materials Research Programs Debut at 1994 MRS Fall Meeting
}

As part of its ongoing effort to keep the MRS Short Course Program relevant and useful to the materials community, the Materials Research Society is pleased to announce two timely additions to the courses and tutorials planned for the 1994 Fall Meeting. A new short course will focus on The Whole Internet: Usage and Futures, and a new tutorial will explore Federal Materials Research Programs and Opportunities.

The Whole Internet: Usage and Futures (A-01): Under President Clinton's goal for a National Information Infrastructure, the Internet becomes the cornerstone of the program and is now available to everyone and for all uses. This short course will introduce participants to the Internet, its roots and culture. The majority of time will be spent examining each of the common network applications. Each tool's use (telnet, ftp, e-mail, news, gopher, archie, and the worldwide web) will be shown and discussed. The full-day course is intended for people with a user's knowledge of computing who are either personally interested in this information resource or are investigating it for their work. No prior knowledge of computer networking is required.

Course date: Wednesday, November 30, 1994, 8:30 a.m. - 4:30 p.m. Instructor: Ed Krol, director, Computing \& Communications Services Office, University of Illinois.

Federal Materials Research Programs and Opportunities (TP-11): Researchers who don't understand the workings of the complex federal materials research programs are at a disadvantage in obtaining support for their ideas. This tutorial will provide a convenient summary of materials science and engineering research programs in federal agencies together with suggested guidance for interacting with and obtaining research support from these agencies. This course will provide an overview of federal agency funding levels, research scope, and directions. It will also cover current opportunities for collaboration and funding and describe the requirements and methodology for obtaining funding. Attendees should leave the course with a enough knowledge to make informed inquiries of the federal agencies or be able to apply directly for support on their own.

Course date: Sunday, November 27, 1994,

8:30 a.m. - 12:00 p.m. Instructor: Louis laniello, consultant, formerly with the U.S. Department of Energy.

See page 84 in this issue for a list of all the courses and tutorials being offered at the 1994 MRS Fall Meeting.

For more information about any of the courses being offered at the 1994 MRS Fall Meeting, or to register while space is still available, contact: Diana L. Schriver, MRS Short Course Manager; phone (412) 3673003, ext. 320; fax (412) 367-4373.

\section{Nominations Being Accepted for MRS Outstanding Young Investigator Award}

Nominations are being accepted for the Materials Research Society's Outstanding Young Investigator Award. This annual award is intended to recognize outstanding, interdisciplinary scientific work in materials research by a young scientist or engineer. The awardee must also show exceptional promise as a developing leader in the materials area. The award consists of a $\$ 1,000$ cash honorarium and a citation plaque. Reasonable travel expenses to attend the MRS meeting at which the award is presented and the meeting registration fee will be reimbursed. Each winner will be invited to present a generalinterest talk to be featured within the structure of the meeting.

\section{Rules and Eligibility}

A nominee must be a young scientist or engineer who has contributed in an outstanding and innovative way to the progress of materials research; the work should have a significant interdisciplinary aspect. The nominee shall not have reached his/her 36th birthday prior to 1 January of the year the award is presented. A nominee need not be a member of the Materials Research Society, and nominees of any national origin or citizenship are eligible.

\section{Nomination Procedure}

A package containing the following is required for nomination for the award:
1. A statement by the nominator supporting the candidate's suitability for the award with respect to:

- innovative and creative nature of the candidate's work,

- interdisciplinary character of the candidate's work, and

- potential shown by the candidate as a future leader in materials research.

2. Supporting information and documents, e.g., curriculum vitae including birth date and a current publication list. Up to three important papers relevant to the award contribution may also be included.

3. Letters of support from two established scientists familiar with the nominee's qualifications and area of research. Only two such letters will be accepted with each nomination. Each letter should make specific reference to the three criteria in item 1 . 4. A list of supporting documents submitted. The entire nomination package, including reprints, should not exceed 10 pages.

Nomination packages for the Outstanding Young Investigator Award to be presented at the 1995 MRS Spring Meeting, should be submitted by October 1, 1994 to: John B. Ballance, Executive Director, Materials Research Society, 9800 McKnight Road, Pittsburgh, PA 152376006.

A nomination for the Outstanding Young Investigator Award remains under consideration for three years, so long as the age requirement for the award is met. The nomination package may be updated by the nominator during that time. MRS

\section{Outstanding Young Investigator Award PREVIOUS RECIPIENTS}

\section{Stuart S.P. Parkin (1991)}

IBM Almaden Research Center

"For ... pioneering contributions to the fields of metallic superlattices, organic superconductors and high-temperature superconductors."

David D. Awschalom (1992)

University of California-Santa Barbara

"For ... pioneering contributions to the physics of molecules in confined geometries, magnetic superlattices, and nanometer-scale magnets."

\section{Charles M. Lieber (1993)}

Harvard University

"For ... pioneering contributions to the understanding of novel materials through synthesis and elegant determination of complex local structure and electronic properties."

\section{David J. Eaglesham (1994)}

AT\&T Bell Laboratories

"For ... experimental ingenuity in the discovery and understanding of fundamental interface, surface, and defect phenomena in semiconductor crystal growth." 